\title{
Computational simulation of safety parameters concerning friction and impact sensitivity of tested civil use explosives
}

\author{
Ilie - Ciprian Jitea ${ }^{1 *}$, Robert Laszlo $^{1}$, Daniela Rus $^{1}$, Ștefan Ilici $^{1}$, and Olga Miclea ${ }^{1}$ \\ ${ }^{1}$ National Institute for Research and Development in Mine Safety and Protection to Explosion - \\ INSEMEX, 32-34 G-ral Vasile Milea St., 332047 - Petroşani, Hunedoara County, Romania
}

\begin{abstract}
The paper presents the results of the theoretical and practical researches regarding the development of conceptual and experimental models specific to the determination of the safety parameters regarding the friction and impact sensitivity, whose plausibility be guaranteed through a whole range of scientific solutions characterized by validated results through numerical modeling and simulations. Designing these types of processes requires consideration, with a relative approximation, the degree of danger due to the design of fragments resulting from detonation, vibrations, pressures, tensions created in the space affected by the explosion, explosion gas generation etc. Estimating the peak values of induced requests is an essential for implementing IT solutions of previous experimentation allows the study of a large number of scenarios, the limit being mainly dependent on the time spent on computer simulations. In this context, safety research into the handling of explosives, the acquisition of experience and knowledge as well as the validation of results by comparison with previous practical experiments and those specified in the literature, become relevant and necessary requirements. Methods used to determine security parameters, analyzed by classical and IT means are relevant to the interpretation of the nature of the explosive matter in terms of their effect in terms of the effect they may have on the environment of use in the event of an explosion in accordance with the legislation in force - an important indicator of classification, handling, storage and transport in the assessment and the management of the risks associated with these operations. The paper presents a series of results regarding computer numerical simulations, safety parameters (friction and impact sensitivity). The similarity of the results obtained in the case of physical experiments to determine the susceptibility of explosive substances tested to friction and impact stimuli in relation to those provided by computerized numerical simulations demonstrate the usefulness of these IT science solutions that through the ANSYS Multiphysics package provide the prerequisites for determining the safety features in the field of explosives for civilian use.
\end{abstract}

\section{Introduction}

\footnotetext{
* Corresponding author: ciprian.jitea@insemex.ro
} 
Research on the use of explosives for civil use in various industrial and civilian applications requires a thorough knowledge of areas such as explosion phenomenology, behaviour of materials in the shock wave, structure dynamics, seismic engineering etc.

The design of these types of processes requires a relative approximation of the degree of danger due to detonation fragments, vibrations, pressures, tensions created in the space affected by the explosion, the generation of explosive gases, etc. The estimation of the maximum values of the induced demands is of crucial importance, the implementation of some a priori experimental solutions allows the study of a large number of scenarios, the limit being mainly dependent on the time spent on computer simulations.

In this context, research in the field, the acquisition of experience and knowledge as well as the validation of results compared to previous practical experiments and those specified in the literature, become relevant and necessary requirements.

Starting from these theoretical and practical considerations, it becomes imperative to focus the research on the development of conceptual and experimental models whose verosimility is guaranteed by a whole series of scientific solutions characterized by validated results through numerical modeling and simulations.

The methods used to determine the ballistic and security parameters analyzed by classical and computer means are relevant to the interpretation of the nature of the explosive matter in terms of its framing in terms of its effect on the environment of use in the event of an explosion according to the legislation in force, being an important indicator of classification, handling, storage and transport in the assessment and management of the risks related to these operations.

- Impact Sensitivity: is the explosive tendency to detonate by impact and is a parameter that characterizes the safety of explosives in handling and transport. It is expressed in joules $(\mathrm{J})$, ie the minimum impact energy at which the explosion of the explosive sample occurs.

- Friction sensitivity: is a parameter that characterizes safety during the manual / mechanized loading of the explosive in mine / well holes, when explosive friction forces can occur between the explosive and the walls of the holes. It is expressed in newton (N) and is the minimum frictional force at which detonations, ignitions or traces of chemical reactions occur.

Due to their specific characteristics, these products are also used in the civilian industry for the execution of works such as: extraction of solid rocks from the massif, in mines or quarries, the execution of mining works (opening galleries) and preparation of mining deposits, tunnels, wells, construction of roads, railways, canals, demolition of foundations / civil / industrial buildings that have become unusable, geophysical-seismometric prospecting etc.

Until detonation under the conditions for which they were carried out, the explosives are at different stages, namely manufacturing, handling, transport, storage. Throughout the course of the explosives, having as their starting point manufacturing and end-use by the operator (authorized personnel), these products may be / are subjected to various demands (friction or impact) that may endanger the life and health of persons and can destroy material goods, affect the environment etc. On this basis, the explosives must have a certain resistance to the demands arising from the handling, storage, loading into the holes and the transport.

Because the types of explosives used in industry have to be related to the nature of the environments in which they will detonate, the knowledge of the parameters characterizing the explosives is essential, whether we refer to the safety of the works in maximum safety conditions or we refer to the parameters thermodynamic, physico-chemical, or ballistics to achieve the proposed objective.

Prior to use (making available on the market), explosives are subject to various laboratory tests to ensure compliance with the essential safety requirements applicable to 
each type and to fit the ballistic / physico-chemical / thermodynamic parameters within specified ranges of explosives specific manufacturers.

In order to evaluate all the parameters characterizing the explosives, there are different methods of verification / determination, most of them being standardized at European / national level, if we refer to the context in which INCD INSEMEX Petroşani is integrated.

The methods currently used for determining friction and impact sensitivity are based on the provisions of harmonized European standards SR EN 13631-3 and SR EN 13631-4. Expression of the results implies a special experience of the research team, the conclusion of the test being reaction / non reaction, at various forces (N) / energies (J) applied on explosive samples based on observed effects such as smoke, explosion noise, flame / spark with or without traces of combustion on substations.

\section{Computerized modeling of the safety parameters of explosives for civil use at the sensitivity to friction and impact}

\subsection{Computerized simulation of the security parameter concerning the sensitivity to friction}

Determination of friction sensitivity at the high explosives and the core of the detonating fuses in order to satisfy the requirements of the Directive 2014/28 / EU, transposed at national level by GD 197/2016 and the Technical Norms to Law no.126 / 1995 with subsequent amendments and normative documents of the products, this test is required.

The method consists in determining a minimum friction force for which it is produced a reaction in eight tests on the fixed sample on a porcelain plate on which it is applied a horizontal translation motion. Friction force is made with a special device for this purpose, through a porcelain pin fixed in the apparatus above the test sample.

For the determination of friction sensitivity, the test was carried out with a granular explosive consisting of a mixture of porous ammonium nitrate and diesel fuel type AUSTINITE manufactured by Austin Powder. The powdered granular substance was passed through a sieve with a mesh of $0.5 \mathrm{~mm}$, for testing using the last fraction that pass this sieve.

For each test was sampled, with a cylindrical measure that have a capacity of $10 \mathrm{~mm} 3$ (2,3 mm diameter and 2,4 $\mathrm{mm}$ length) a sample of the prepared substance according to the working procedure.

\subsection{The test procedure}

The sample of the test material measured with the apparent volume unit of $10 \mathrm{~mm}^{3}$ shall be placed on the porcelain plate, fixed on the slide of the apparatus in such a way that the striations of the plate are perpendicular to the direction of movement of the table.

The pin is fasten in the arm of the device.

The sample is placed on the porcelain plate so that the pin stays partially on the sample and some of it is in front of the pin in the direction of movement.

The rod arm is carefully leave in a horizontal position for the application of force on the sample to be tested, by rod suspension in the last notch using the weight of $10 \mathrm{~kg}$, resulting in a $360 \mathrm{~N}$ friction force. The actuator button of the apparatus is press and send a single backward motion to the sample, the behaviour of the sample is recorded, either as a positive result in the case of a reaction $(\mathrm{R})$ or without reaction (FR). 
Because it was not found a reaction to the explosive sample (FR) in the case of a $360 \mathrm{~N}$ load, the test was repeated 5 times, and after the observation of the mode of display of the explosive samples tested, no reactions were found.

\subsection{Calculation, expression and evaluation of results}

Friction sensitivity is expressed by the minimum frictional force (Newton) at which a reaction occurs in six (six) consecutive tests to determine the friction sensitivity of explosives. The value of the friction force $(\mathrm{N})$ is determined by the mass used and its position on the appliance stem during the test.

The test method does not imply a calculation of uncertainty because the result is of a qualitative type, respectively "with reaction" (R) or "without reaction" (FR). Following the test for determining the friction sensitivity of the granular explosive consisting of a mixture of porous ammonium nitrate and diesel, type AUSTINITE, resulted in a friction force value greater than $360 \mathrm{~N}$ (Table 1).

Table 1. Test results for AUSTINITE

\begin{tabular}{|c|c|c|c|}
\hline $\begin{array}{c}\text { No. } \\
\text { crt. }\end{array}$ & $\begin{array}{c}\text { The frictional force } \\
{[\mathbf{N}]}\end{array}$ & $\begin{array}{c}\text { Room temperature } \\
{\left[{ }^{\mathbf{0}} \mathbf{C}\right]}\end{array}$ & $\begin{array}{c}\text { Result: } \\
\text { R-reaction; } \\
\text { FR - without reaction }\end{array}$ \\
\hline $\mathbf{1}$ & 360 & 22 & FR \\
\hline $\mathbf{2}$ & 360 & 22 & FR \\
\hline $\mathbf{3}$ & 360 & 22 & FR \\
\hline $\mathbf{4}$ & 360 & 22 & FR \\
\hline $\mathbf{5}$ & 360 & 22 & FR \\
\hline $\mathbf{6}$ & 360 & 22 & FR \\
\hline
\end{tabular}

\subsection{Computerized simulation of friction sensitivity}

Thus, by using the Geometry module within the ANSYS Multiphysics package, the geometry of the porcelain plate and the explosive charge placed on its surface was realized.

Through the ANSYS Workbench platform, the connection between the Geometry module and the Explicit Dynamics application was made, in which the discretization network for the entire virtual model was created in order to perform the numerical simulation of the friction sensitivity of the explosive consisting of a mixture of ammonium nitrate porous and diesel, type AUSTINITE (figure 1). As can be seen from the figure, the discretization is highly refined in the area of interest, respectively in the area of the explosive sample placement, in order to obtain qualitative and quantitative results with increased accuracy. At the same time, the contact surfaces between the interacting elements were defined: explosive, porcelain pin, as well as the defining characteristics of these elements: explosive type AUSTINITE and pin (masses, positions of mass placement on the device rod, etc) [1].

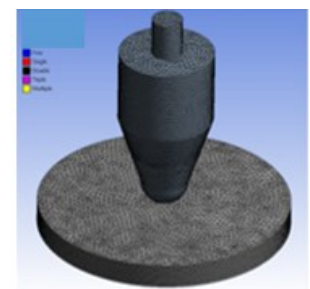

Fig.1 Discretization for the elements of the numerical simulation model for friction sensitivity determination. 
The result obtained, respectively, the finding of the absence of a reaction (FR) in the mass of explosive substance tested, following the application of a load of $360 \mathrm{~N}$ (corresponding to the use of a mass of $10 \mathrm{~kg}$ located at a distance of $360 \mathrm{~mm}$, respectively on the last notch of the rod of the apparatus Iulius Peter used for the test) is presented in Figure 2.

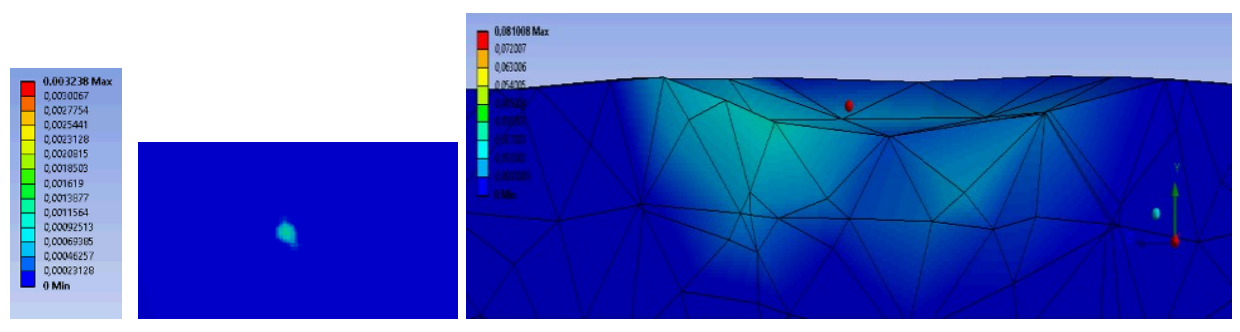

Fig.2 The finding of the absence of a reaction (FR) in the mass of explosive substance tested, following the application of a load of $360 \mathrm{~N}$.

This result is the same as the one obtained in the case of the physical experiment $(360$ $\mathrm{N}$ ), which demonstrates the usefulness of computer simulations using the ANSYS Multiphysics package in the field of explosives for civil use for determining the safety parameter regarding friction sensitivity.

\subsection{Computerized simulation of the security parameter regarding the impact sensitivity}

Thus, by using the Geometry module within the ANSYS Multiphysics package, the geometry of the impact device and the explosive load placed within it.

Through the ANSYS Workbench platform, the connection was made between the Geometry module and the Explicit Dynamics application, in which the geometry of the ensemble was realized, the discretization network for the entire virtual model in order to perform the numerical simulation of the impact sensitivity of the explosive consisting of a mixture of porous ammonium nitrate and diesel fuel type AUSTINITE (figures 3, 4, 5 and 6) on 2 computers a number of 4990 finished volumes comprising 5480 knots). As can be seen from the figure, the discretization is highly refined in the area of interest, respectively the inside of the impact device where the explosive sample is located, in order to obtain qualitative and quantitative results with increased accuracy. At the same time, the contact surfaces between the interacting elements were defined: explosive, intermediate anvil as well as the defining characteristics of these elements: explosive type AUSTINITE and intermediate anvil(masses, falling heights, etc.). In the Figures 7 and 8 are highlighted the diagrams with the evolution of kinetic energy parameters and the component on the $\mathrm{Y}$ axis of the impulse force $[2,3,4]$. 


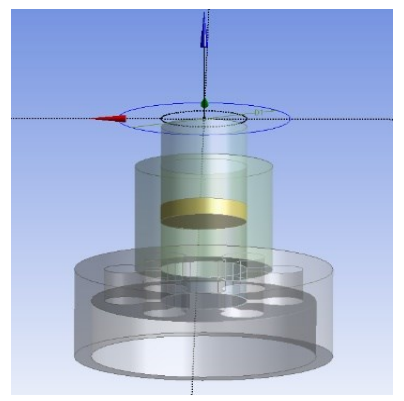

Fig.3 The geometry of the assembly.

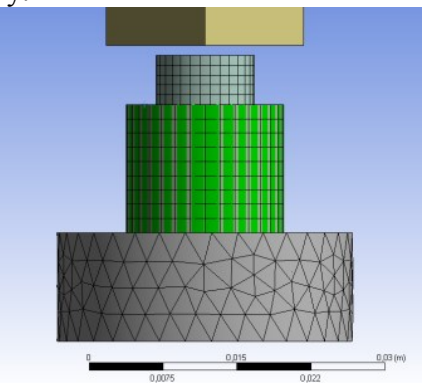

Fig.4- Discretization for the elements of the numerical simulation model of the impact sensitivity determination.

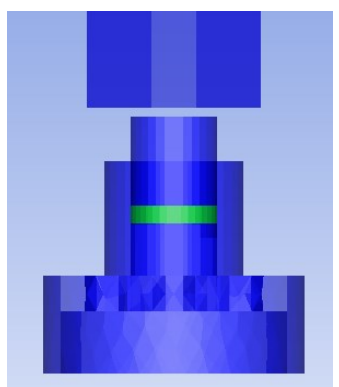

Fig.5- Image taken from AUTODYN before impact is triggered.

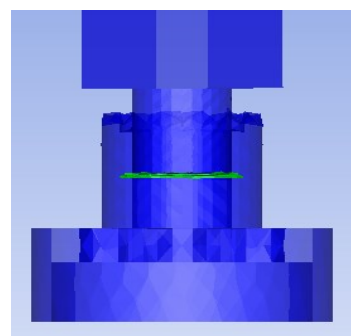

Fig.6- The image taken from AUTODYN after the impact trigger. 
Material Summary ( Ident 0 - admodel )

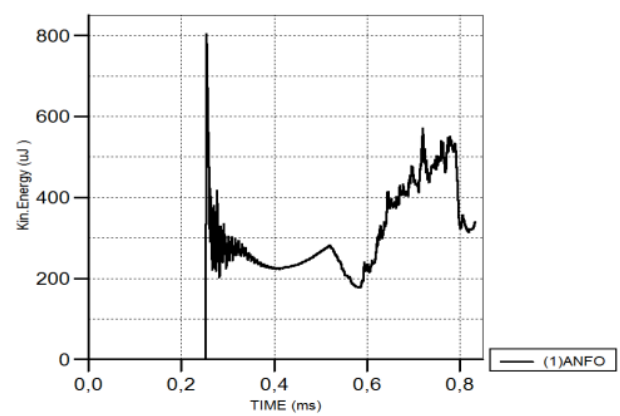

Material Summary ( Ident 0 - admodel )

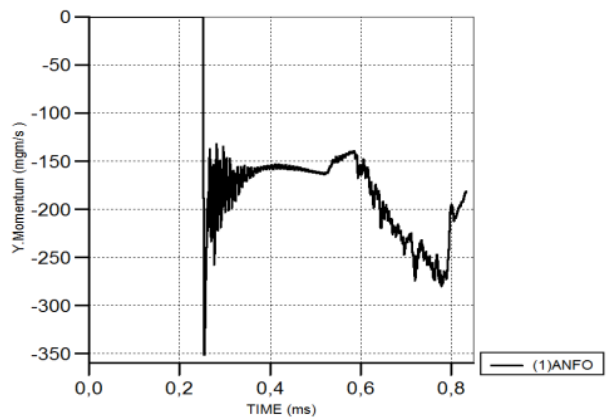

Fig.7- The kinetic energy of the impact.

Fig.8- The component of the impulse force on the y axis.

After setting the main characteristics of the elements of the virtual model, in compliance with the values used in the physical experiment - "Determining the impact sensitivity", the running of the Explicit Dynamics application using the AUTODYN consisted of: computer 1 (33150 calculation cycles, on a workstation with 12 processors - CORES); computer 2 (60945 calculation cycles, on a workstation with 30 processors - CORES). The result obtained did not lead to the finding of any reaction $(\mathrm{R})$ in the mass of explosive substance tested (apart from the pressure deformation of the quantity of explosive substance under test), due to the generation of impact energy of approximately $40.0 \mathrm{~J}$ corresponding to the drop of a mass of $5 \mathrm{~kg}$ from a height of $65 \mathrm{~cm}$, under the conditions of taking into account a gravitational acceleration of $10 \mathrm{~m} / \mathrm{s}^{2}$. This result leads to the idea of research continuing in the field of computer applications to obtain a virtual model capable of reproducing the conditions for determining the safety parameter regarding the impact sensitivity of explosives for civil use, using the ANSYS Multiphysics package.

Designing these types of processes requires considering, with a relative approximation, the degree of danger due to the design of fragments resulting from the detonation, vibrations, pressures, tensions created in the space affected by the explosion, generation of explosion gases, etc. The estimation of the maximum values of the induced requests being of an essential importance, the implementation of computer solutions of a priori experimentation allows the study of a large number of scenarios, the limit being mainly dependent on the time allotted to the computer simulations. In this context, research in the field, the acquisition of experience and knowledge as well as the validation of the results in comparison with previous practical experiments and those specified in the specialized literature, become relevant and necessary requirements.

Starting from these theoretical and practical considerations, it becomes imperative to focus research on the development of conceptual and experimental models, whose plausibility is guaranteed by a whole series of scientific solutions characterized by results validated through numerical modelling and simulation.

In order to develop the IT infrastructure in the field of evaluating the security parameters (sensitivity to friction and impact) specific to the explosives for civilian use, the methodologies for determining these characteristics by classical and informational means were analysed, in order to make conceptual and experimental models whose verisimilitude is guaranteed by scientific solutions characterized by results validated through numerical modelling and simulations using the ANSYS Multiphysics software package and the Explicit Dynamics specialized application with the AUTODYN solver..

This paper was developed within the NUCLEU - Programme, carried out with the support of ANCSI, Project no. PN-19 210202. 


\section{References}

1. IC. Jitea, C. Lupu, M. Suvar, D. Rus, Environmental Engineering and Management Journal 13, 1459-1462 (2014)

2. E. Ghicioi, IC. Jitea, NI. Vlasin, A.Kovacs, G. Vasilescu, E. Gheorghiosu, M. Prodan, $14^{\text {th }}$ GeoConference on Science and Technologies in Geology, Exploration and Mining, Albena, Bulgaria, 139-145 (2014)

3. VM. Pasculescu, NI. Vlasin, MC. Suvar, GD. Florea, Z. Vass, $18^{\text {th }}$ GeoConference on Science and Technologies in Geology, Exploration and Mining, Albena, Bulgaria, 4754 (2018)

4. GD. Vasilescu, A. Doru, B. Florin, B. Crinela, O. Miclea, $18^{\text {th }}$ GeoConference on Science and Technologies in Geology, Exploration and Mining, Albena, Bulgaria, 785-792 (2018) 